\title{
Bistability and self-pulsation in quantum-dot lasers with intracavity quantum-dot saturable absorbers
}

\author{
O. Qasaimeh, W.-D. Zhou, J. Phillips, S. Krishna, and P. Bhattacharya ${ }^{\text {a) }}$ \\ Solid-State Electronics Laboratory, Department of Electrical Engineering and Computer Science, \\ University of Michigan, Ann Arbor, Michigan 48109-2122
}

M. Dutta

Army Research Office, Research Triangle Park, North Carolina 27709

(Received 6 November 1998; accepted for publication 16 January 1999)

\begin{abstract}
Self-organized $\mathrm{In}_{0.4} \mathrm{Ga}_{0.6} \mathrm{As} / \mathrm{GaAs}$ quantum-dot single-mode ridge waveguide lasers with intracavity absorber were grown by molecular beam epitaxy. Bistability in the light-current characteristics of $3 \mu \mathrm{m}$ single-mode edge-emitting laser was obtained by controlling the intracavity absorber voltage. Self-pulsation was also observed with a center frequency of $1.6 \mathrm{GHz}$ and linewidth $<10 \mathrm{MHz}$. (C) 1999 American Institute of Physics. [S0003-6951(99)00912-2]
\end{abstract}

Room-temperature self-organized quantum-dot lasers have demonstrated very high differential gain, ${ }^{1,2}$ low threshold current, ${ }^{1}$ temperature insensitivity of the threshold current over wide ranges of temperature, ${ }^{1}$ and small-signal modulation bandwidth exceeding $20 \mathrm{GHz}^{2}$ Vertical cavity surface emitting lasers (VCSELs) with quantum-dot gain regions have also been recently demonstrated. ${ }^{3}$ The unique gain and absorption characteristics of quantum dots arising from the near-singular density of states and the large differential gain are expected to influence other dynamic characteristics of the device, such as self-pulsations, mode locking, and chirp. ${ }^{1}$ Controlled self-pulsation in a laser eliminates the need for high-frequency drive circuitry and optical feedback is also reduced or eliminated due to the short coherence length of the devices. It is expected that the higher exciton binding energy and lower effective temperature of carriers in the quasizero dimensional system will enable better phasespace filling in a saturable absorber. The carrier recombination times, which control the recovery time in a saturable absorber, are also smaller than in quantum wells. ${ }^{4}$ In this letter, we demonstrate bistability and self-pulsation in selforganized edge-emitting quantum-dot laser with integrated intracavity absorber region formed with the same quantum dots.

Growth of the $\mathrm{In}_{0.4} \mathrm{Ga}_{0.6} \mathrm{As} / \mathrm{GaAs}$ five-quantum-dot (QD) layer laser heterostructure, shown in Fig. 1, was done by molecular beam epitaxy. Details of such growth have been described elsewhere. ${ }^{5}$ The $\mathrm{In}_{0.4} \mathrm{Ga}_{0.6} \mathrm{As}$ QD regions, grown in the three-dimensional Stranski-Krastanow growth mode, are separated by $15 \AA$ of GaAs barriers. The dot density, as measured by atomic force microscopy, is $5 \times 10^{10} \mathrm{~cm}^{-2}$. The near pyramidal dots are $14 \mathrm{~nm}$ in base length and $7 \mathrm{~nm}$ in height. The rest of the separate confine heterostructure (SCH) laser consists of appropriate inner and outer cladding layers and contact regions.

The laser and the intracavity saturable absorber device were fabricated in the ground-signal-ground (GSG) configuration by using standard photolithography and lift-off techniques and a combination of dry and wet etching. The device is shown schematically in Fig. 1. The gap between the gain

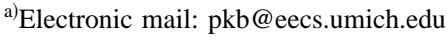

and absorber sections is $5 \mu \mathrm{m}$ in width and extends almost to the inner cladding layer above the quantum dots. The laser and absorber single-mode waveguides are also etched to the same depth. The deep etch in the gap ensures a resistance larger than $2 \mathrm{k} \Omega$ between the two sections. The width of the waveguides is $3 \mu \mathrm{m}$ and the lengths $l_{L}$ and $l_{A}$ as indicated in Fig. 1 varies from 340 to $400 \mu \mathrm{m}$ and 15 to $60 \mu \mathrm{m}$, respectively. $p$ - and $n$-type ohmic metallization were done with $\mathrm{Pd} / \mathrm{Zn} / \mathrm{Pd} / \mathrm{Au}$ and $\mathrm{Ni} / \mathrm{Ge} / \mathrm{Au} / \mathrm{Ti} / \mathrm{Au}$, respectively. A thick $\mathrm{SiO}_{2}$ layer formed by plasma-enhanced chemical vapor deposition is used for device passivation and isolation. 1.4 $\mu \mathrm{m} \mathrm{Ti} / \mathrm{Al} / \mathrm{Ti} / \mathrm{Au}$ was finally evaporated to form the interconnects. Biasing of the device was done with a standard coplanar microwave probe.

The lasing wavelength in the devices was observed to be $1.01 \mu \mathrm{m}$. Measured pulsed light-current $(L-I)$ characteristics are shown in Fig. 2 for $l_{L}=400 \mu \mathrm{m}$ and $l_{A}=60 \mu \mathrm{m}$. The measurements were made with different values of saturable

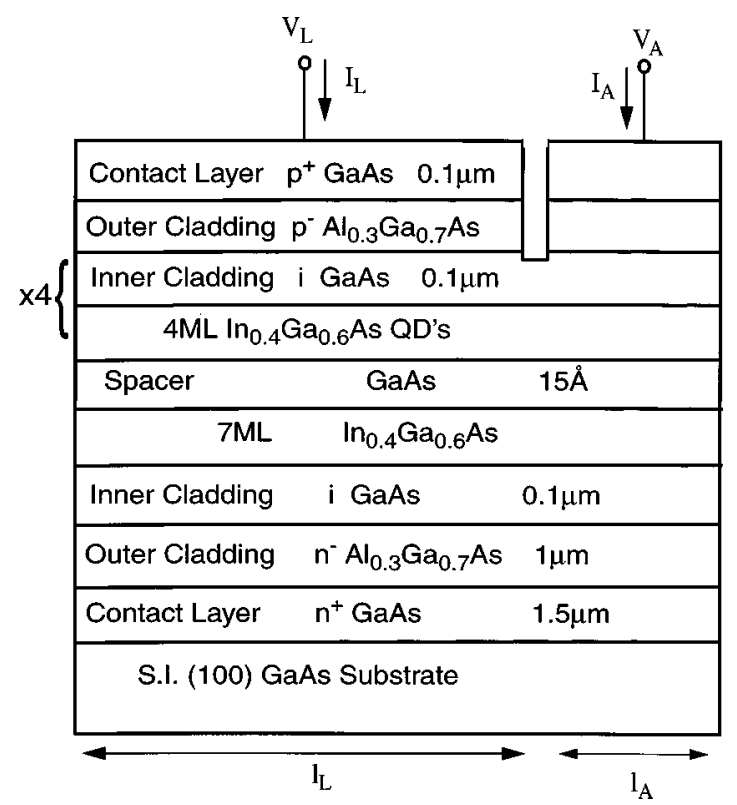

FIG. 1. Heterostructure for quantum-dot laser and intracavity absorber grown by molecular beam epitaxy, where $l_{L}$ and $l_{A}$ are the laser and the absorber lengths, respectively, $I_{L}$ is the laser current, and $V_{A}$ is the absorber applied voltage. 


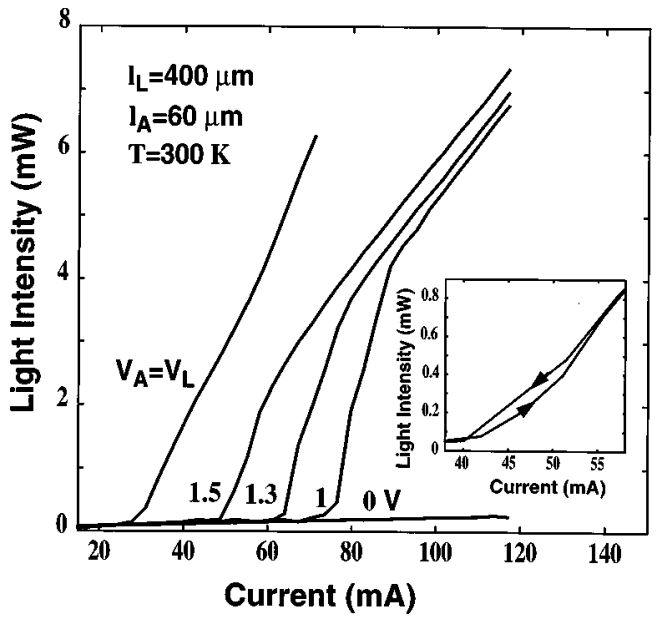

FIG. 2. Measured room-temperature light-current $(L-I)$ characteristics of the device with $l_{L}=400 \mu \mathrm{m}$ and $l_{A}=60 \mu \mathrm{m}$. The inset shows bistability in the $L-I$ characteristics of a device having $l_{L}=400 \mu \mathrm{m}, l_{A}=50 \mu \mathrm{m}$, and $V_{A}=1 \mathrm{~V}$.

absorber (SA) voltage $V_{A}$. As can be seen, the laser has a threshold of $26 \mathrm{~mA}$ when the SA region is shorted to the gain region. Increasing the reverse bias of the SA increases the cavity losses and the laser responds with a higher threshold corresponding to a higher gain region of the quantum dots. This change of the threshold current with SA voltage is linear and is attributed to the nonsaturable loss in the absorber. ${ }^{6}$ A distinct discontinuity in the slope of the $L-I$ curves, observed at higher output powers, is attributed to a saturable loss arising from nonlinear absorption phenomena in the quantum dots. We have also observed bistability in the $L-I$ characteristics, as shown in the expanded inset of Fig. 2, for specific lengths of the SA and voltage applied to it. The data shown in the inset of Fig. 2 are with a device having $l_{L}=400 \mu \mathrm{m}, l_{A}=50 \mu \mathrm{m}$ and $V_{A}=1 \mathrm{~V}$.

In order to understand the origin of the bistability, we examined the absorber current-voltage $(I-V)$ characteristics for different values of laser bias current. Typical SA $I-V$ characteristics are shown in Fig. 3. When the absorber is forward biased, it behaves as a normal diode. Under reverse bias, the absorber current at a constant laser current is determined by two competing processes: the field, which increases the current, and the increased absorption, which re-

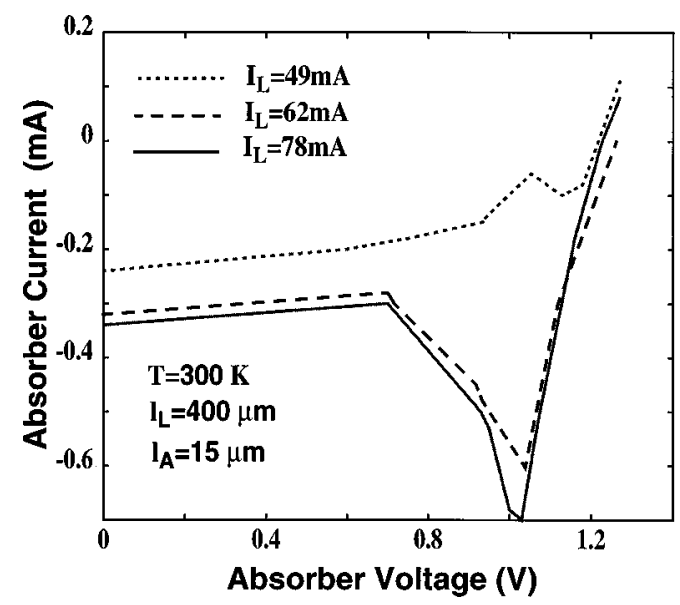

FIG. 3. Room-temperature voltage-current characteristics of the absorber for different values of laser bias current.

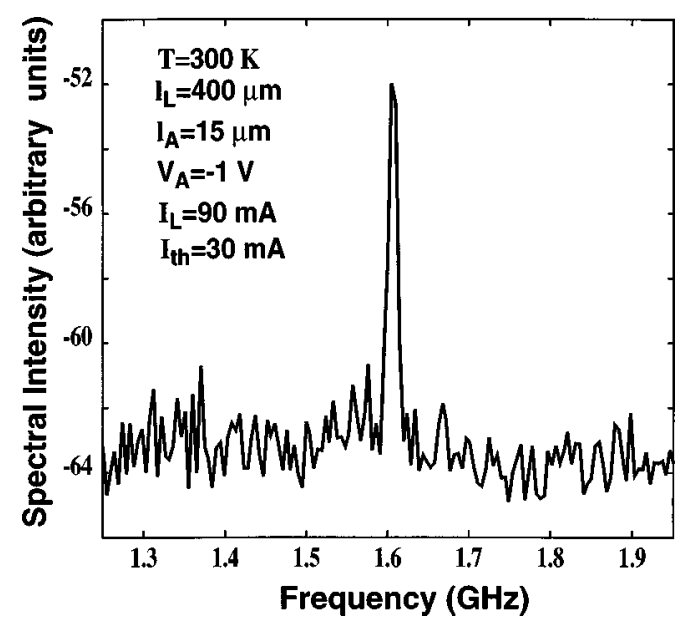

FIG. 4. rf spectral density of self-pulsation in quantum-dot laser with intracavity absorber at room temperature.

duces the laser power and consequently the absorber current. It is assumed that a red shift of the absorption peak of the QD occurs with increasing bias. At low reverse bias the former process dominates. At higher bias, the latter dominates, resulting in a negative differential resistance (NDR) in the characteristic, as shown in Fig. 3. At higher values of the bias, the large absorption prevents lasing altogether and the SA current increases again. The bistability in the $L-I$ characteristics occurs when the load line corresponding to the series resistance of the SA bias circuit intersects the SA $I_{A}-V_{A}$ characteristics three times. Similar phenomena have been observed in quantum well devices. ${ }^{7}$

Self-pulsation phenomena were measured using a highspeed $(40 \mathrm{GHz})$ Newfocus photodetector and a $22 \mathrm{GHz}$ Hewlett-Packard radio frequency (rf) spectrum analyzer. The trace shown in Fig. 4 was obtained with $I_{L}=90 \mathrm{~mA}$ and $V_{A}=-1 \mathrm{~V}$. The self-pulsation is centered at $1.6 \mathrm{GHz}$ with a rf linewidth $(-3 \mathrm{~dB})$ of $<10 \mathrm{MHz}$. Self-pulsation was obtained over a fairly narrow range of $V_{A}(0.9-1.4 \mathrm{~V})$ and this depends on the bias load line and the NDR characteristics of the SA. Since these can be controlled, to some extent, the self-pulsation can be used for reliable high-frequency sources.

For potential applications in communication and microwave systems, it is of interest to explore high-frequency mode locking in QD devices. Mode locking can be achieved, without self-pulsation, at very large intracavity photon density and low threshold with high reflectivity coatings and large absorber length. We are currently investigating these aspects.

In summary, we have demonstrated self-pulsation and bistability in a quantum-dot laser integrated with a quantumdot saturable absorber. Self-pulsations are characterized by a center frequency of $1.6 \mathrm{GHz}$ and linewidth $<10 \mathrm{MHz}$.

This work is being supported by the Army Research Office under Grant No. DAAG55-9710156.

${ }^{1}$ D. Bimberg, N. Kirstaedter, N. Ledentsov, ZH. Alferov, P. Kopev, and V. Ustinov, IEEE J. Sel. Top. Quantum Electron. 3, 196 (1997).

${ }^{2}$ D. Klotzkin, K. Kamath, K. Vineberg, P. Bhattacharya, R. Murty, and J. Laskar, IEEE Photonics Technol. Lett. 10, 932 (1998).

${ }^{3}$ D. Huffaker, O. Baklenov, L. Graham, B. Streetman, and D. Deppe, Appl. Phys. Lett. 70, 2356 (1997) 
${ }^{4}$ K. Kamath, N. Chervela, K. Linder, T. Sosnowski, H-T. Jiang, T. Norris, J. Singh, and P. Bhattacharya, Appl. Phys. Lett. 71, 927 (1997).

${ }^{5}$ K. Kamath, P. Bhattacharya, T. Sosnowski, T. Norris, and J. Phillips, Electron. Lett. 32, 1374 (1996).
${ }^{6}$ J. O'Gorman, A. Levei, R. Nottenburg, T. Tanbun-EK, and R. Logan, Appl. Phys. Lett. 57, 968 (1990).

${ }^{7}$ S. Lim, J. Hudgings, G. Li, W. Yuen, K. Lau, and C. Chang-Hasnain, Electron. Lett. 33, 1708 (1997). 